\title{
Activity pacing for osteoarthritis symptom management: study design and methodology of a randomized trial testing a tailored clinical approach using accelerometers for veterans and non-veterans
}

Susan L Murphy ${ }^{1,2^{*}}$, Angela K Lyden ${ }^{1}$, Maria Clary², Michael E Geisser ${ }^{1}$, Raymond L Yung ${ }^{2,3}$, Daniel J Clauw ${ }^{4}$ and David A Williams ${ }^{4}$

\begin{abstract}
Background: Osteoarthritis $(\mathrm{OA})$ is a prevalent chronic disease and a leading cause of disability in adults. For people with knee and hip OA, symptoms (e.g., pain and fatigue) can interfere with mobility and physical activity. Whereas symptom management is a cornerstone of treatment for knee and hip OA, limited evidence exists for behavioral interventions delivered by rehabilitation professionals within the context of clinical care that address how symptoms affect participation in daily activities. Activity pacing, a strategy in which people learn to preplan rest breaks to avoid symptom exacerbations, has been effective as part of multi-component interventions, but hasn't been tested as a stand-alone intervention in OA or as a tailored treatment using accelerometers. In a pilot study, we found that participants who underwent a tailored activity pacing intervention had reduced fatigue interference with daily activities. We are now conducting a full-scale trial.

Methods/Design: This paper provides a description of our methods and rationale for a trial that evaluates a tailored activity pacing intervention led by occupational therapists for adults with knee and hip OA. The intervention uses a wrist accelerometer worn during the baseline home monitoring period to glean recent symptom and physical activity patterns and to tailor activity pacing instruction based on how symptoms relate to physical activity. At 10 weeks and 6 months post baseline, we will examine the effectiveness of a tailored activity pacing intervention on fatigue, pain, and physical function compared to general activity pacing and usual care groups. We will also evaluate the effect of tailored activity pacing on physical activity (PA).

Discussion: Managing OA symptoms during daily life activity performance can be challenging to people with knee and hip OA, yet few clinical interventions address this issue. The activity pacing intervention tested in this trial is designed to help people modulate their activity levels and reduce symptom flares caused by too much or too little activity. As a result of this trial, we will be able to determine if activity pacing is more effective than usual care, and among the intervention groups, if an individually tailored approach improves fatigue and pain more than a general activity pacing approach.
\end{abstract}

Trial Registration: ClinicalTrials.gov: NCT01192516

\footnotetext{
* Correspondence: sumurphy@umich.edu

'Department of Physical Medicine and Rehabilitation, University of Michigan,

Ann Arbor, MI, USA

Full list of author information is available at the end of the article
} 


\section{Background}

Osteoarthritis (OA) is a prevalent and chronic disease. It affects over 27 million adults in the U.S. and is expected to increase as the population ages [1]. OA is more prevalent among females, however, male veterans are also significantly affected, with $1 / 3$ of males of all ages diagnosed and nearly half of those who use the Veteran's Affairs (VA) healthcare system [2]. OA causes substantial physical and psychosocial disability. By 2020, it is expected that 12 million people in the U.S. will report activity limitations due to arthritis [3].

A primary reason people with OA seek treatment is for pain relief. Whereas people with OA often receive pharmacological treatment for pain reduction, nonpharmacological treatment for symptoms is considered the foundation of OA management [4,5]. Rehabilitation professionals, such as occupational therapists (OTs), can tailor non-pharmacological symptom management interventions so as to promote adherence and behavior change. To date, however OTs have had only limited involvement in tailoring such interventions for people with OA. More research is needed on the effectiveness of these tailored symptom management interventions when delivered by rehabilitation professionals within the context of clinical care. Well-established communitybased programs on self-management strategies (such as the Arthritis Self-Management Program) have been shown to have small but positive effects on pain and arthritis self-efficacy [6-8]. Although community-based programs are an important tool for OA management, such programs do not optimize the use of rehabilitation professionals in the delivery or tailoring of these programs.

\section{Behavior Change and Tailoring Interventions}

A tailored approach to interventions is being increasingly recommended in order to make the content personally relevant and to promote long-term behavior change. Several interventions are individually tailored on key variables identified in theoretical health behavior models, such as motivational readiness, decisional balance, or self-efficacy [9-11]. Interventions also have been tailored on personal characteristics (e.g., gender [12], depression [13], pain level [14]). There is growing evidence that tailoring enhances the effectiveness of clinical interventions designed to alter health risk behaviors such as smoking $[15,16]$ and low physical activity $[17,18]$.

For arthritis management interventions, Social Cognitive Theory has often been used as the theoretical foundation of intervention development $[19,20]$. It posits that people learn through an interaction of social and environmental influences that contribute to their own motivations and behaviors [21]. To adopt a new health behavior, the central determinants include 1) knowledge of the risks and benefits, 2) outcome expectations about costs and benefits for engaging in health behaviors, 3 ) the health goals people set for themselves and plans for goal achievement, 4) the perceived facilitators and barriers (such as external social or work demands), and 5) self-efficacy, the belief in the ability to perform under a certain set of conditions [21,22]. Self-efficacy is often a central focus in self-management interventions because it influences behavior both directly and indirectly through the other determinants above [22]. Numerous studies have found that improvements in pain coping skills are related to improvements in arthritis self-efficacy $[19,23,24]$.

In this study, the intervention is designed to assist people in enhancing their arthritis self-efficacy by individually tailoring the use of activity pacing strategies to the context of where and when the strategies may be most useful. According to Social Cognitive Theory, selfefficacy is enhanced by building competency through practice (and by the rewards associated with success) in specific situations or conditions [22]. By tailoring activity pacing instruction to individuals' real-life routines and activities, there is a greater chance for learning to occur and for self-efficacy to be enhanced given the greater proximity of the pacing schedule to real-life and personally relevant activities.

\section{Pain, Fatigue, and Their Relationship to Physical Activity}

Both pain and fatigue symptoms are linked to various functional problems in OA. Pain in OA affects the ability to engage in activities of daily living, work, and other meaningful activities and is associated with a reduced quality of life [25-27]. Fatigue is not as well-studied in $\mathrm{OA}$, however, it is one of the most frequently reported OA symptoms $[28,29]$. Attention to the clinical importance of fatigue is growing. In large studies, substantial fatigue was reported by $41 \%$ of patients with OA [28], and problematic fatigue or tiredness was reported in moderate levels [30]. Fatigue in OA is one of the strongest predictors of functional disability $[28,31]$ and work dysfunction [28]. Fatigue has also been associated with pain, sleep problems, functional disability, and depression [28,30-32]. Qualitative studies have further characterized the fatigue experience in $\mathrm{OA}$ as debilitating, causing activity restriction, and greatly impacting life $[29,33]$.

A better understanding of how symptoms affect activity on a more micro level, such as over a day, week, or month, can help refine and tailor OA interventions. For example, tailored interventions may target timing of behaviors such as medication usage or variability in symptoms by time [34]. Although OA symptoms are believed to be less variable than other rheumatic 
conditions such as rheumatoid arthritis (RA), an emerging body of research has shown that there is important variability in OA pain [35-38] across days or even within a day. These pain fluctuations have been associated with limited daily activities, days of missed work, sleep interference, and mood [36], and increased pain variability may be associated with personal factors [e.g., body mass index (BMI)] [35].

Fewer studies have examined fatigue variability in OA. Given the strong relationship between pain and fatigue $[28,31,32]$ in OA and other conditions, it is not surprising that fatigue is often considered a by-product of pain. However, when pain and fatigue are measured daily or within-day, differences emerge. For example, compared to samples of persons with RA and fibromyalgia, daily fatigue fluctuations were not as strongly associated with daily pain fluctuations in persons with OA [39]. In our work, we found that within-day fatigue was experienced at greater intensities than was pain [40]. Additionally, in one of the few studies to examine the symptom-activity relationship in OA, we found that fatigue was more highly associated with objective physical activity (PA; measured by accelerometer) compared to pain [40]. Lastly, we found differential effects for pain and fatigue by group when we tailored activity pacing [41], providing support for addressing pain and fatigue separately.

\section{Pacing Intervention Effectiveness}

Although activity pacing is a recommended non-pharmacological intervention for OA management based on consensus guidelines [4], its effectiveness as a standalone intervention is understudied. Typically, activity pacing is combined with other programs (e.g., coping skills training, cognitive behavioral therapy). In these studies of knee or hip OA, the overall programs have been effective at reducing pain [23,24]; however, it is impossible to disentangle the unique effects of activity pacing. Due to differences in how activity pacing is understood as a strategy, there is variability in activity pacing instruction and its delivery by therapists $[42,43]$. This trial addresses these limitations by using a standardized approach to teaching activity pacing to adults with knee or hip OA. This approach is structured around the overall goal of activity pacing, which is the balance between activity and rest in order to accomplish necessary and valued activities [44]. In this study, we will also be able to examine the unique effects of tailored activity pacing on fatigue for knee or hip OA in which our preliminary results showed significant improvements in fatigue compared to a general activity pacing intervention [41]. The conceptual model that guided intervention development is shown in Figure 1.

\section{Methods/Design}

This is a three-arm randomized controlled trial that will examine outcomes at baseline, posttest (approximately 10 weeks post baseline), and 6 months. Ethical approval for this study was obtained by the University of Michigan Medical School Institutional Review Board and the Subcommittee on Human Studies in the Veteran's Affairs Ann Arbor Healthcare System. The overall goal of the study is to examine the effectiveness of a tailored activity pacing intervention for adults with symptomatic knee or hip OA. The primary outcomes are fatigue and pain.

The specific aims are:

Aim 1: To examine the effectiveness of a tailored activity pacing intervention on fatigue, pain, and physical function.

Aim 2: To determine if increased arthritis self-efficacy post intervention is related to improvements in symptom severity and function.

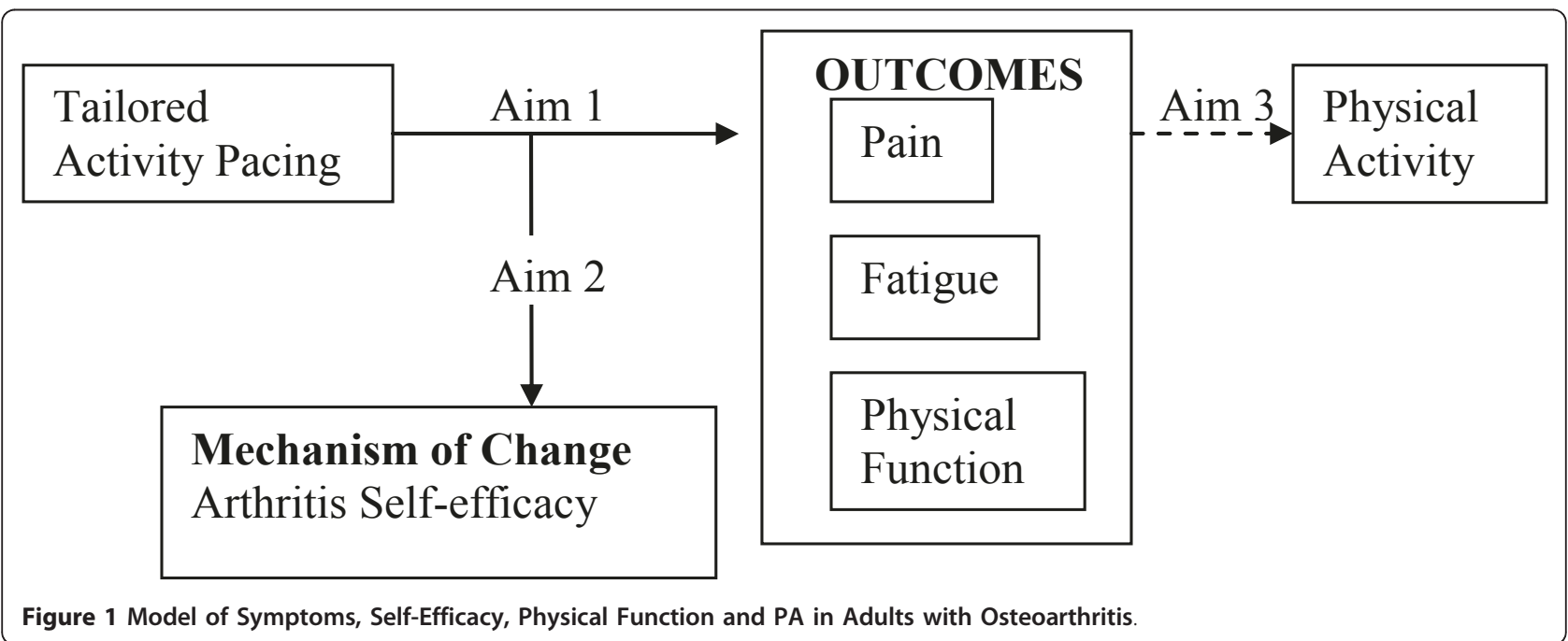


Aim 3: To evaluate the effect of tailored activity pacing on physical activity.

Eligible individuals are randomly stratified by age and gender into one of three arms: tailored activity pacing intervention, general activity pacing intervention, or usual care. The tailored and general activity pacing interventions are equivalent in session number and length. Participants are involved in study procedures over 6 months. For the intervention groups, involvement consists of 3 individual sessions, 3 testing visits, 3 home monitoring periods, 3 weekly follow-up phone calls, and monthly contact phone calls until the outcome assessment at 6 months. The usual care group participates in all activities described above except for the intervention sessions and the 3 weekly follow-up phone calls.

\section{Participant Recruitment}

Community-living veteran and non-veteran participants are recruited from the University of Michigan and VA clinics, senior housing sites, and through public advertisements. Based on our previous studies, our optimal recruitment methods are ad placement at clinics and through community ads and fliers. As in our previous studies, areas with higher concentrations of minorities are particularly targeted for recruitment. Minorities such as African Americans are particularly important to include in this study because they not only have higher rates of knee and hip OA than Caucasians [45], they also report more severe OA symptoms and more physical disability even when controlling for demographics and other factors [46]. In addition, African Americans are less likely than Caucasians to opt for hip or knee replacements [47] and more likely to manage OA through home remedies that often have little to no evidence of effectiveness (such as copper bracelets or herbal medicines) [48].

\section{Eligibility Criteria}

The following inclusion criteria were designed to identify a cohort of community-living adults who are likely experiencing symptoms specifically due to their OA. Prior to enrollment, each prospective participant is evaluated for inclusion. Table 1 lists the inclusion and exclusion criteria.

\section{Procedures}

Figure 2 shows a flow chart of the study protocol. In brief, trained research personnel screen individuals for eligibility, administer performance-based assessments, and train participants in the use of the wrist-worn accelerometer and accompanying logbook. Participants provide written consent to participate in the study. They wear the accelerometer for a 7 day period and then

\section{Table 1 Eligibility Criteria}

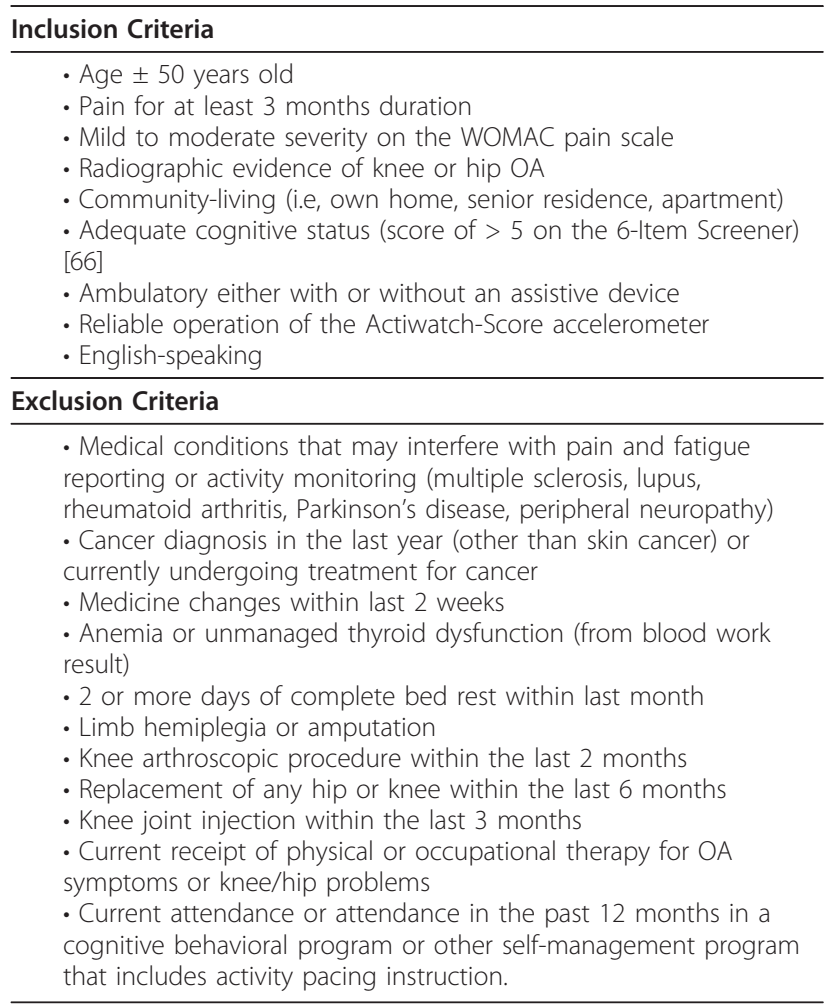

return materials in person or by mail to the study team. Participants are randomly stratified by age and gender into the tailored activity pacing intervention, general activity pacing intervention, or usual care group. The usual care group participants undergo all outcome assessments and have maintained contact through monthly emails between outcome assessments similar to the intervention groups. The contact period between Weeks $11-23$ is being undertaken to better ensure participation in the 6 month assessment for all groups. Each intervention arm involves 3 individual sessions with an OT. In the tailored activity pacing group, data collected from the home monitoring period are used to generate reports for participants about how symptoms relate to physical activity and are used by the occupational therapist. The general activity pacing group does not use the information from the home monitoring period during OT sessions.

\section{Study Arms \\ Usual Care Group}

This group participates in all outcome measures and monthly outcomes are tracked during the study period using a survey measure to determine if any new OA pharmacological or non-pharmacological treatments are undertaken (e.g., injection, physical therapy, medication change) to treat symptoms. 


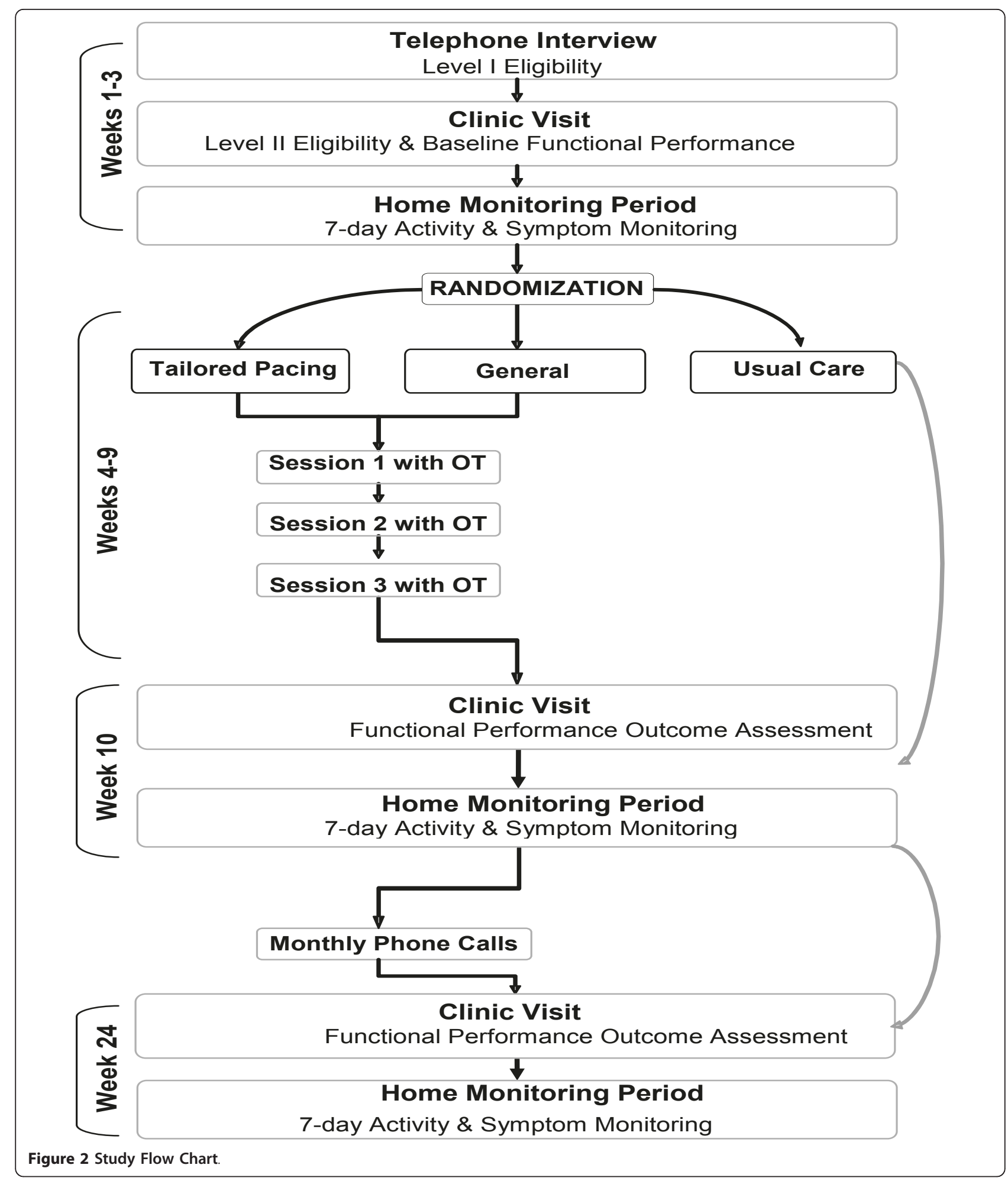

General and Tailored Activity Pacing Interventions

Regardless of whether people are assigned to the tailored activity pacing or general activity pacing intervention groups, participants meet with an OT for 3 individual sessions (total treatment time: 3 hours over Weeks 4-9 of the study). The first session for both the tailored activity pacing and general activity pacing includes a brief presentation by the OT on the nature of 
OA and how physical demand and mood can affect the symptom experience. This is followed by group-specific instruction on activity pacing as a tool for symptom management which includes printed materials that are a take-home reference for the participant. In the tailored activity pacing intervention, the OT discusses how to incorporate activity pacing based on the individual's profile of symptoms and activities determined from the baseline home monitoring period. In the general activity pacing intervention, the OT uses generic examples and does not mention the individual symptom experience from the baseline home monitoring period. By the end of the first OT session for both the tailored and general activity pacing groups, the participants develop a timebased activity plan with goals related to activity pacing. The second session focuses on individual progress with activity pacing, goal setting, and addressing barriers to using the recommended strategies. The third session focuses on reviewing activity pacing tenets, evaluating the use of time based activity pacing and planning for the future. After the 3 individual sessions have concluded, the OT makes 3 brief follow-up phone calls (1 call per week) to discuss activity pacing progress, symptoms, and any barriers.

\section{Strategies for Ensuring Treatment Fidelity}

Treatment fidelity is defined as "the procedures used to monitor and enhance the reliability and validity of behavioral interventions [49]," and is an essential component of this study. Per recommendations from the National Institutes of Health (NIH) behavior change consortium $[49,50]$, several strategies are being used to ensure treatment fidelity in this study. These strategies target fidelity of treatment delivery (standardized modules for the intervention and interventionist training and monitoring) and treatment receipt (i.e., participant involvement in treatment).

\section{Fidelity of Treatment Delivery}

We have designed the study such that both intervention groups are equivalent in dose and administration of treatment (i.e., the number and length of sessions), including the manner in which participants are contacted from 10 weeks through 6 months. There are several procedures in place to ensure fidelity in how treatment is delivered. It is likely that there will be multiple therapists per active intervention arm (i.e., tailored activity pacing and general activity pacing). We decided that extra therapists per group could reduce the possibility of treatment effect due to therapist characteristics and ensure that we can deliver the intervention in the event that any interventionists drop out of the study. All therapists will undergo an initial training period which will involve structured education via a manual and a series of didactic sessions with the principal investigator (PI) and study team members. The initial training will also involve role playing in pairs and an observer who will rate the mock treatment session for key concepts to ensure consistency across therapists. Any discrepancies in how the intervention is delivered across therapists will be discussed and a consensus will be reached about intervention delivery.

To assess therapist adherence to the intervention protocol, they are asked to log the actual treatment time per session with each individual. The therapist also has a checklist of items for each visit to facilitate consistent presentation of the protocol. In addition, we are audiotaping sessions (as long as each individual consents to that process) and an independent rater listens to the tapes shortly after the sessions to determine if original criteria for intervention delivery in each treatment arm are being met. Any discrepancies in the audiotapes by therapists in a particular treatment arm will be resolved in a face-to-face meeting through consensus to reduce any variability in delivery. To prevent protocol 'drift', the PI and therapists in each arm have monthly telephone contact to discuss any issues and additional training sessions among therapists occurs as needed.

\section{Fidelity of Treatment Receipt}

We will also assess participant factors related to treatment fidelity, i.e., how the treatment is received by the participant. This involves an assessment of individual factors such as comprehension of information, and the ability to use and perform skills associated with both the data collection and behavioral elements of the study. We have a standardized interactive learning module to teach the participant how to input symptoms on the wristworn accelerometer. Because the validity of the symptom information is central to our tailoring methods, all participants will undergo this learning module prior to their first session with the OT. In addition, both the tailored and general activity pacing interventions include strategies to ensure comprehension of the material. For example, participants are asked to reiterate main pacing concepts back to the therapist (e.g., importance of planning, resting before symptoms get worse, etc.). Participants also receive homework assignments that reinforce the lessons presented during the session and are asked to complete a daily activity logbook. Both of these homework items are used to assess personal compliance and overall protocol adherence. Each subsequent session involves a review of previously presented topics, a review of goals, and time for discussion of any barriers. Session 3 is focused on relapse-prevention, and continues the theme of setting goals and problem-solving to minimize barriers that impede the use of activity pacing. Lastly, we conduct debriefing interviews with each 
participant to examine their experiences in the intervention including adherence with activity pacing in daily life at the end of their involvement in the study.

\section{Measures}

The primary outcome measures are fatigue and pain. Fatigue is assessed in two ways. First, fatigue is measured by the Brief Fatigue Inventory (BFI) [51], a reliable and valid measure of fatigue in adults with cancer. There is no gold standard measure of fatigue for adults with OA; however, the BFI is of particular relevance because it assesses fatigue by asking about interference in daily life, which is our primary interest. This scale is usually scored by calculating an overall score based on an average of 9 of the 10 items. In samples with rheumatic conditions, the BFI has recently been divided into two fatigue subscales (fatigue severity and fatigue interference) [52]. Although no studies have examined the clinical significance of these subscales in OA or in other samples, we are expecting similar or greater improvements in fatigue severity and fatigue interference as seen in our pilot study ( $8 \%$ and $15 \%$ improvement respectively) [41]. Second, because there is currently no commonly-used fatigue measure in OA across studies, an additional method of assessing fatigue from the PatientReported Outcomes Measurement (PROMIS) assessment tools will be used. PROMIS is a collection of carefully constructed item banks that can be used to assess multiple domains of relevance to complex and chronic illnesses. Due to the methods used to construct these item banks, such measures are expected to be more precise and less burdensome to patients to complete [53]. We are using the 8-item short form PROMIS fatigue scale [54]. Pain, the other primary outcome, is assessed by the Western Ontario and McMaster Universities Osteoarthritis Index (WOMAC). This is a validated, disease-specific questionnaire for adults with knee or hip OA in which participants rate their currently experienced pain and physical disability [55]. Based on previous studies, we will consider a 2 point decrease on the WOMAC pain scale to be clinically significant [56].

Other secondary outcomes and potential moderators include physical function, assessed by the 6 Minute Walk Test [57], a commonly used, validated measure of physical performance in adults; arthritis self-efficacy, the perceived ability to manage arthritis and pain and fatigue symptoms, measured by the Arthritis Self Efficacy Scale [58]; and other variables such as knee extension strength (as measured by a hand-held dynamometer), and general health status variables (e.g., BMI, selfreported chronic conditions, depression using the Geriatric Depression Scale [59], medication use). In addition, PA will be assessed by a wrist-worn accelerometer [Actiwatch-Score, Phillips Respironics-Mini Mitter Co, Bend
OR] that measures changes in acceleration. Although it is worn on the wrist, it is highly associated with wholebody movement $[60,61]$. Changes in acceleration are recorded as activity counts and saved every 15 seconds. Higher activity counts reflect participation in higher intensity activities [62,63]. PA from the accelerometer will be aggregated in different ways. We will look at average activity counts and total activity counts that occur: 1) in the time between symptom reporting periods, 2) over each day, and 3) over the entire home monitoring period similar to our previous studies $[40,41,64]$.

\section{Procedure for the Home Monitoring Periods}

The accelerometer is mounted on each participant's non-dominant wrist. Participants are instructed on how to enter responses into the accelerometer using a standardized interactive learning module that we have developed and used in previous studies. Participants are given the opportunity to practice rating their symptoms and using the accelerometer's input button to record the information. If, for some reason, participants fail the learning module (e.g., inability to rate symptom experience on a numeric rating scale, inability to press the input button) they are excluded. Although we have not encountered exclusion for this reason in any of our past studies, this reporting is central to the tailoring portion of the intervention and therefore is an important criterion for study inclusion. Participants also become familiar with the logbook that accompanies the accelerometer and serves to cross-validate the items. The logbook is used as a back-up if there are missing data from the accelerometer.

Momentary pain and fatigue severity are measured on 0 -10 numerical rating scales that will be directly input into the accelerometer five times a day (at rise time in the morning, 3 times during waking hours, and at bedtime). The accelerometer is worn for 7 days at baseline and at the outcome assessment periods. Pain is rated on a scale from $0=$ no pain to $10=$ pain as bad as you can imagine. Fatigue, defined as tiredness or weariness [28], is rated on a scale from $0=$ no fatigue to $10=$ fatigue as bad as you can imagine. Momentary ratings are used in the study for the sole purpose of generating tailored reports on individual symptom-activity relationships during the baseline week for the tailored activity pacing intervention.

\section{Data Analysis}

The target sample size in this study is 156 participants. In our recruitment plans, we have factored in a $30 \%$ attrition rate to achieve this number. Although there are three arms to the study: tailored activity pacing, general activity pacing, and usual care group, the study was powered to find effects between the two active 
intervention groups (tailored activity pacing and general activity pacing). Specifically, the power analysis was based on our pilot data in which we compared the tailored activity pacing intervention with the general activity pacing intervention and detected large group differences using the effect size Cohen's d [65] on BFI fatigue severity and interference $(\mathrm{d}=.79$ and 1.1 respectively) at 10 week-follow-up. Based on a 2 group t-test at a significance level of $\mathrm{p}=.05$, we will have greater than $90 \%$ power to detect an effect size of .74 or larger with 40 people in each of the two active intervention groups. Because most people with OA do not receive this type of intervention, it is important to compare the active intervention groups to a group receiving usual care. The study was powered to detect treatment effects between the active intervention groups; therefore we will also have sufficient power to detect differences between the active intervention groups and usual care group.

To examine the effectiveness of a tailored activity pacing intervention on fatigue, pain, and physical function, we will use repeated measures analysis of variance (RM ANOVA) models to analyze the responses (pain, fatigue, and 6 minute walk test) at baseline, 10 weeks, and 6 months for the three treatment groups (tailored activity pacing, general activity pacing, and usual care group). This will allow us to estimate the effects of treatment group, time point and the treatment $\times$ time interaction. Similarly, we will use RM ANOVA models to determine if increased arthritis self-efficacy post intervention is related to improvements in symptom severity and function and to evaluate the effect of tailored activity pacing on physical activity.

\section{Discussion}

Although non-pharmacological treatment is recommended for people with knee and hip OA to manage symptoms, much of the evidence-base has examined these strategies in the context of a program of skills rather than individually. This study is examining the effect of a brief, individually-tailored, OT-led activity pacing intervention on pain and fatigue in adults with knee and hip OA. Given the positive effects of the tailored activity pacing approach compared to general activity pacing on fatigue in a small pilot study, we hypothesize that fatigue in particular will be improved with the tailored activity pacing intervention. We will also explore arthritis self-efficacy as a potential mechanism of change and the effects of pacing on PA at the 6 month follow-up period in an exploratory analysis.

\section{Acknowledgements and Funding}

All participants provide written informed consent to participate in this study. This study was funded through a grant from the VA Rehabilitation Research and Development Service (IRX000410A). We thank Kathy Welch for her statistical consultation on this project.

\section{Author details}

'Department of Physical Medicine and Rehabilitation, University of Michigan, Ann Arbor, MI, USA. VA Ann Arbor Healthcare System, GRECC, Ann Arbor, MI, USA. ${ }^{3}$ Department of Internal Medicine, Division of Geriatric Medicine, University of Michigan, Ann Arbor, MI, USA. ${ }^{4}$ Department of Anesthesiology, University of Michigan, Ann Arbor, MI, USA.

\section{Authors' contributions}

SLM, AKL, MEG, RLY, DJC, and DAW have made substantial contributions to conception and design of this study. SLM and AKL made significant contributions to acquisition of data, the data analysis plan, and interpretation of data. All authors have been involved in drafting the manuscript or revising it critically for important intellectual content. All authors have given final approval of the version to be published.

\section{Competing interests}

The authors declare that they have no competing interests.

Received: 14 July 2011 Accepted: 2 August 2011

Published: 2 August 2011

\section{References}

1. Lawrence R, Felson D, Helmick C, Arnold L, Choi H, Deyo R, Gabriel S, Hirsch R, Hochberg M, Hunder G, Jordan JM, Katz JN, Kremers HM, Wolfe F: Estimates of the prevalence of arthritis and other rheumatic conditions in the United States. Part II. Arthritis Rheum 2008, 58:26-35.

2. Dominick KL, Golightly YM, Jackson GL: Arthritis prevalence and symptoms among US non-veterans, veterans, and veterans receiving Department of Veterans Affairs Healthcare. J Rheumatol 2006, 33:348-354.

3. Arthritis prevalence and activity limitations-United States 1990. MMWR Morb Mortal Wkly Rep 1994, 43:433-438.

4. Zhang W, Moskowitz RW, Nuki G, Abramson S, Altman RD, Arden N, Bierma-Zeinstra S, Brandt KD, Croft P, Doherty M, Dougados M, Hochberg M, Hunter DJ, Kwoh K, Lohmander LS, Tugwell P: OARSI recommendations for the management of hip and knee osteoarthritis, part I: critical appraisal of existing treatment guidelines and systematic review of current research evidence. Osteoarthritis Cartilage 2007, 15:981-1000.

5. Dieppe PA, Lohmander LS: Pathogenesis and management of pain in osteoarthritis. Lancet 2005, 365:965-973.

6. Superio-Cabuslay E, Ward MM, Lorig KR: Patient education interventions in osteoarthritis and rheumatoid arthritis: a meta-analytic comparison with nonsteroidal antiinflammatory drug treatment. Arthritis Care Res 1996, 9:292-301

7. Warsi A, Wang P, LaValley M, Avorn J, Solomon D: Self-management education programs in chronic disease: a systematic review and methodological critique of the literature. Arch Intern Med 2004, 164:1641-1649.

8. Zhang W, Nuki G, Moskowitz RW, Abramson S, Altman RD, Arden NK, Bierma-Zeinstra S, Brandt KD, Croft P, Doherty M, Dougados M, Hochberg M, Hunter DJ, Kwoh K, Lohmander LS, Tugwell P: OARSI recommendations for the management of hip and knee osteoarthritis: Part III: changes in evidence following systematic cumulative update of research published through January 2009. Osteoarthritis Cartilage 2010, 18:476-499.

9. Kerns RD, Rosenberg R: Predicting responses to self-management treatments for chronic pain: application of the pain stages of change model. Pain 2000, 84:49-55.

10. Marcus $B H$, Forsyth $\mathrm{LH}$ : Tailoring interventions to promote physically active lifestyles in women. Womens Health Issues 1998, 8:104-111.

11. Shin $Y$, Hur $H$, Pender $N$, Jang $H$, Kim MS: Exercise self-efficacy, exercise benefits and barriers, and commitment to a plan for exercise among Korean women with osteoporosis and osteoarthritis. Int J Nurs Stud 2006, 43:3-10.

12. Theis K, Helmick C, Hootman J: Arthritis burden and impact are greater among U.S. women than men: intervention opportunities. J Womens Health 2007, 16:441-453. 
13. Damush T, Wu J, Bair M, Sutherland J, Kroenke K: Self-management practices among primary care patients with musculoskeletal pain and depression. J Behav Med 2008, 31:301-307.

14. Turk DC, Rudy TE, Kubinski JA, Zaki HS, Greco CM: Dysfunctional patients with temporomandibular disorders: evaluating the efficacy of a tailored treatment protocol. J Consult Clin Psychol 1996, 64:139-146.

15. Strecher V, Wang C, Derry H, Wildenhaus K, Johnson C: Tailored interventions for multiple risk behaviors. Health Educ Res 2002, 17:619-626.

16. Prochaska JO, DiClemente CC, Velicer WF, Rossi JS: Standardized, individualized, interactive, and personalized self-help programs for smoking cessation. Health Psychol 1993, 12:399-405.

17. Greaney M, Riebe D, Garber C, Rossi J, Lees F, Burbank P, Nigg C, Ferrone C, Clark P: Long-term effects of a stage-based intervention for changing exercise intentions and behavior in older adults. Gerontologist 2008, 48:358-367.

18. van Sluijs EMF, van Poppel MNM, van Mechelen W: Stage-based lifestyle interventions in primary care: are they effective? Am J Prev Med 2004, 26:330-343.

19. Lorig KR, Mazonson PD, Holman HR: Evidence suggesting that health education for self-management in patients with chronic arthritis has sustained health benefits while reducing health care costs. Arthritis Rheum 1993, 36:439-446.

20. Hughes SL, Seymour RB, Campbell R, Pollak N, Huber G, Sharma L: Impact of the fit and strong intervention on older adults with osteoarthritis. Gerontologist 2004, 44:217-228.

21. Bandura A: Self-Efficacy: The Exercise of Control.Edited by: Freeman WH 1997.

22. Bandura A: Health promotion by social cognitive means. Health Educ Behav 2004, 31:143-164.

23. Keefe FJ, Caldwell DS, Williams DA, Gil KM, Mitchell D, Robertson C, Martinez S, Nunley J, Beckham JC, Crisson JE, Helms M: Pain coping skills training in the management of osteoarthritic knee pain: a comparative study. Behav Ther 1990, 21:49-62.

24. Keefe FJ, Caldwell DS, Williams DA, Gil KM, Mitchell D, Robertson C, Martinez S, Nunley J, Beckham JC, Helms M: Pain coping skills training in the management of osteoarthritic knee pain-Il: follow-up results. Behav Ther 1990, 21:435-447.

25. Hill CL, Parsons J, Taylor A, Leach G: Health related quality of life in a population sample with arthritis. J Rheumatol 1999, 26:2029-2035.

26. Boutron I, Rannou F, Jardinaud-Lopez M, Meric G, Revel M, Poiraudeau S: Disability and quality of life of patients with knee or hip osteoarthritis in the primary care setting and factors associated with general practitioners' indication for prosthetic replacement within 1 year Osteoarthritis Cartilage 2008, 16:1024-1031.

27. Grotle M, Hagen K, Natvig B, Dahl F, Kvien T: Prevalence and burden of osteoarthritis: results from a population survey in Norway. $J$ Rheumatol 2008, 35:677-684.

28. Wolfe F, Hawley DJ, Wilson K: The prevalence and meaning of fatigue in rheumatic disease. J Rheumatol 1996, 23:1407-1417.

29. Power JD, Badley E, French M, Wall A, Hawker G: Fatigue in osteoarthritis: a qualitative study. BMC Musculoskelet Disord 2008, 9:63.

30. Sale JEM, Gignac M, Hawker G: The relationship between disease symptoms, life events, coping and treatment, and depression among older adults with osteoarthritis. J Rheumatol 2008, 35:335-342.

31. Wolfe F: Determinants of WOMAC function, pain and stiffness scores: evidence for the role of low back pain, symptom counts, fatigue and depression in osteoarthritis, rheumatoid arthritis and fibromyalgia. Rheumatology 1999, 38:355-361.

32. Fishbain D, Cole B, Cutler RB, Lewis J, Rosomoff HL, Fosomoff RS: Is pain fatiguing? A structured evidence-based review. Pain Med 2003, 4:51-62.

33. Gignac M, Davis A, Hawker G, Wright J, Mahomed N, Fortin P: "What do you expect? You're just getting older": A comparison of perceived osteoarthritis-related and aging-related health experiences in middleand older-age adults. Arthritis Rheum 2006, 55:905-912.

34. Allen $\mathrm{K}$ : The value of measuring variability in osteoarthritis pain. $J$ Rheumatol 2007, 34:2132-2133.

35. Allen KD, Coffman CJ, Golightly YM, Stechuchak KM, Keefe FJ: Daily pain variations among patients with hand, hip, and knee osteoarthritis. Osteoarthritis Cartilage 2009, 17:1275-1282.
36. Affleck G, Tennen H, Keefe FJ, Lefebvre JC, Kashikar-Zuk S, Wright K, Starr K, Caldwell DS: Everyday life with osteoarthritis or rheumatoid arthritis: independent effects of disease and gender on daily pain, mood, and coping. Pain 1999, 83:601-609.

37. Hutchings A, Calloway M, Choy E, Hooper M, Hunter DJ, Jordan JM, Zhang Y, Baser O, Long S, Palmer L: The longitudinal examination of arthritis pain (LEAP) study: relationships between weekly fluctuations in patient-related joint pain and other health outcomes. J Rheumatol 2007, 34:2291-2300.

38. Keefe F, Affleck G, France C, Emery C, Waters S, Caldwell D, Stainbrook D, Hackshaw K, Fox L, Wilson K: Gender differences in pain, coping, and mood in individuals having osteoarthritic knee pain: a within-day analysis. Pain 2004, 110:571-577.

39. Zautra AJ, Fasman R, Parish BP, Davis MC: Daily fatigue in women with osteoarthritis, rheumatoid arthritis, and fibromyalgia. Pain 2007 128:128-135

40. Murphy SL, Smith DM, Clauw DJ, Alexander NB: The impact of momentary pain and fatigue on physical activity in women with osteoarthritis. Arthritis Rheumatism 2008, 59:849-856

41. Murphy S, Lyden A, Smith D, Dong Q, Koliba J: Effects of a tailored activity pacing intervention on pain and fatigue for older adults with osteoarthritis. Am J Occup Ther 2010, 64:869-876.

42. Gill J, Brown C: A structured review of the evidence for pacing as a chronic pain intervention. Eur J Pain 2009, 13:214-216

43. Beissner K, Henderson C, Papaleontiou M, Olkhovskaya Y, Wigglesworth J, Reid MC: Physical therapists' use of cognitive-behavioral therapy for older adults with chronic pain: a nationwide survey. Phys Ther 2009, 89:456-469.

44. Murphy SL, Clauw DJ: Activity pacing: what are we measuring and how does it relate to treatment? Pain 2010, 149:582-583.

45. Golightly YM, Dominick KL: Racial variations in self-reported osteoarthritis symptom severity among veterans. Aging Clin Exp Res 2005, 17:264-269.

46. Satish S, Postigo LG, Ray LA, Goodwin JS: Chronic rheumatologic symptoms in a tri-ethnic sample of men and women aged 75 and older. J Gerontol A Biol Sci Med Sci 2001, 56:M471-M476.

47. Skinner J, Weinstein J, Sporer S, Wennberg J: Racial, ethnic, and geographic disparities in rates of knee arthroplasty among Medicare patients. N Engl J Med 2003, 349:1350-1359.

48. Ibrahim SA, Siminoff LA, Burant CJ, Kwoh CK: Variation in perceptions of treatment and self-care practices in elderly with osteoarthritis: a comparison between African American and white patients. Arthritis Rheum 2001, 45:340-345

49. Bellg A, Borrelli B, Resnick B, Hecht J, Minicucci D, Ory M, Ogedegbe G, Orwig D, Ernst D, Czajkowski S: Enhancing treatment fidelity in health behavior change studies: best practices and recommendations from the NIH Behavior Change Consortium. Health Psychol 2004, 23:443-451.

50. Borrelli B: The assessment, monitoring, and enhancement of treatment fidelity In public health clinical trials. J Public Health Dent 2011, 71 S52-S63.

51. Mendoza TR, Wang XS, Cleeland CS, Morrissey M, Johnson BA, Wendt JK, Huber SL: The rapid assessment of fatigue severity in cancer patients: use of the Brief Fatigue Inventory. Cancer 1999, 85:1186-1196.

52. Wolfe F: Fatigue assessments in rheumatoid arthritis: comparative performance of visual analog scales and longer fatigue questionnaires in 7760 patients. J Rheumatol 2004, 31:1896-1902.

53. Cella D, Riley W, Stone A, Rothrock N, Reeve B, Yount S, Amtmann D, Bode R, Buysse D, Choi S, Cook K, Devellis R, DeWalt D, Fries JF, Gershon R, Hahn EA, Lai JS, Pilkonis P, Revicki D, Rose M, Weinfurt K, Hays R, PROMIS Cooperative Group: The Patient-Reported Outcomes Measurement Information System (PROMIS) developed and tested its first wave of adult self-reported health outcome item banks: 2005-2008. J Clin Epidemiol 2010, 63:1179-1194

54. The Assessment Center. [http://www.assessmentcenter.net]

55. Bellamy N, Buchanan WW, Goldsmith $\mathrm{CH}$, Campbell J, Stitt LW: Validation study of WOMAC: a health status instrument for measuring clinically important patient relevant outcomes to antirheumatic drug therapy in patients with osteoarthritis of the hip or knee. J Rheumatol 1988, 15:1833-1840.

56. Farrar JT, Portenoy RK, Berlin JA, Kinman JL, Strom BL: Defining the clinically important difference in pain outcome measures. Pain 2000 , 88:287-294. 
57. Butland RJ, Pang J, Gross ER, Woodcock AA, Geddes DM: Two-, six-, and 12-minute walking tests in respiratory disease. British Medical Journal Clinical Research Ed 1982, 284:1607-1608.

58. Lorig K, Chastain RL, Ung E, Shoor S, Holman HR: Development and evaluation of a scale to measure perceived self-efficacy in people with arthritis. Arthritis Rheum 1989, 32:37-44.

59. Yesavage JA, Brink TL, Rose TL, Lum O, Huang V, Adey M, Leirer VO: Development and validation of a geriatric depression screening scale: a preliminary report. J Psychiatr Res 1982, 17:37-49.

60. Patterson SM, Krantz DS, Montgomery LC, Deuster PA, Hedges SM, Nebel LE: Automated physical activity monitoring: validation and comparison with physiological and self-report measures. Psychophysiology 1993, 30:296-305.

61. Westerterp KR: Physical activity assessment with accelerometers. Int $J$ Obes Relat Metab Disord 1999, 23:S45-S49.

62. Swartz AM, Strath SJ, Bassett DR, O'Brien WL, King GA, Ainsworth BE: Estimation of energy expenditure using CSA accelerometers at hip and wrist sites. Med Sci Sports Exerc 2000, 32:S450-S456.

63. Murphy SL: Review of physical activity measurement using accelerometers in older adults: considerations for research design and conduct. Prev Med 2009, 48:108-114.

64. Murphy SL, Strasburg DM, Lyden AK, Smith DM, Koliba JF, Dadabhoy DP, Wallis SM: Effects of activity strategy training on pain and physical activity in older adults with knee or hip osteoarthritis: a pilot study. Arthritis Rheum 2008, 59:1480-1487.

65. Cohen J: Statistical Analyses for the Behavioral Sciences. Hillsdale: Lawrence Erlbaum Associates; 21988.

66. Callahan C, Unverzagt F, Hui S, Perkins A, Hendrie H: Six-item screener to identify cognitive impairment among potential subjects for clinical research. Med Care 2002, 40:771-781.

\section{Pre-publication history}

The pre-publication history for this paper can be accessed here: http://www.biomedcentral.com/1471-2474/12/177/prepub

doi:10.1186/1471-2474-12-177

Cite this article as: Murphy et al: Activity pacing for osteoarthritis symptom management: study design and methodology of a randomized trial testing a tailored clinical approach using accelerometers for veterans and non-veterans. BMC Musculoskeletal Disorders 2011 12:177.

\section{Submit your next manuscript to BioMed Central and take full advantage of:}

- Convenient online submission

- Thorough peer review

- No space constraints or color figure charges

- Immediate publication on acceptance

- Inclusion in PubMed, CAS, Scopus and Google Scholar

- Research which is freely available for redistribution

Submit your manuscript at www.biomedcentral.com/submit
C Biomed Central 